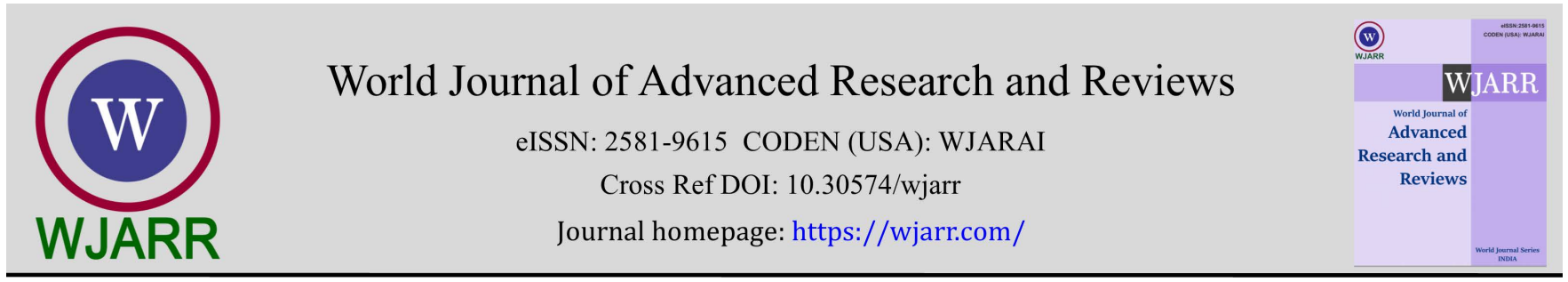

(CASE REPORT)

Check for updates

\title{
Hemiplegia revealing an abscessed pituitary adenoma: A case report
}

Fresnel Lutèce ONTSI OBAME 1, ${ }^{*}$, Alngar DJIMRABEYE 1, Yao Christian Hugues DOKPONOU 2, Moussa Elmi SAAD ${ }^{1}$, Nourou Dine Adeniran Bankole ${ }^{1}$, Adyl MELHAOUI ${ }^{1}$ and Abdessamad EL OUAHABI ${ }^{1}$

${ }^{1}$ Department of Neurosurgery, Hôpital des spécialités de Rabat, Mohammed V University of Rabat - Morocco.

2 Department of Neurosurgery, Mohammed V Military Teaching Hospital, Rabat - Morocco.

World Journal of Advanced Research and Reviews, 2022, 13(01), 351-354

Publication history: Received on 07 December 2021; revised on 10 January 2022; accepted on 12 January 2022

Article DOI: https://doi.org/10.30574/wjarr.2022.13.1.0027

\begin{abstract}
Abscesses secondary to pituitary adenoma are rare. The common clinical manifestations often encountered are headaches, meningismus, and visual disturbances. Hemiplegia as its revealing sign is uncommon. To the best of our knowledge, this is the second reported case of a pituitary abscess with a focal neurological deficit of the half-sided body. We report a case of a 52-year-old woman successfully treated by stereotactic evacuation of pituitary abscess inside an aggressive pituitary tumor. The pituitary abscess must be suspected early for adequate management because it is a serious complication.
\end{abstract}

Keywords: Pituitary Abscess; Aggressive Adenoma; Hemiplegia; Stereotaxis; Clinical Case

\section{Introduction}

Pituitary adenomas are the most common benign tumors associated with the pituitary gland. These are slow-growing tumors that arise from the cells of the gland [1]. Aggressive pituitary tumors (APT) are recognized by the scientific community for the existence of a higher risk of recurrence, invasion of parasellar structures, and resistance to multimodal therapies. The prevalence of APT is unclear [2]. Abscess of pituitary adenoma (PA) is a rare pathological entity with high morbidity and mortality [2,3]. Clinical manifestations common to pituitary abscesses are chronic headache, visual disturbances, and symptoms of pituitary insufficiency [3]. But it is very rare to have a patient presenting with hemiplegia secondary to an abscess developed in the pituitary adenoma.

We report a case of a 52-year-old woman admitted with right-sided hemiplegia revealing an abscessed aggressive pituitary macroadenoma. We will discuss the available diagnosis tools and management in light of the literature.

\section{Patient and observation}

A 52-year-old female patient was admitted for progressive headaches of variable intensity associated with right side body weakness ongoing for the past two weeks. Her past medical history is respectively a left mastectomy for breast cancer with adjuvant chemotherapy in February 2017; an aggressive pituitary macroadenoma diagnosed by microsurgical biopsy using a trans-sphenoidal approach in May 2017 and treated with gamma knife radiosurgery; and a ventriculoperitoneal shunt in 2019 for hydrocephalus. She has been in blindness since 2017.

Neurological examination revealed dysarthria and 1/5 right side hemiplegia. There was no meningism. The patient had no sensory deficit and her body temperature was $38^{\circ} \mathrm{C}$.

\footnotetext{
${ }^{*}$ Corresponding author: Fresnel Lutèce ONTSI OBAME

Department of Neurosurgery, Hôpital des spécialités de Rabat, Mohammed V University of Rabat - Morocco

Copyright $(2022$ Author(s) retain the copyright of this article. This article is published under the terms of the Creative Commons Attribution Liscense 4.0.
} 
The MRI brain showed a circumscribed mass with suprasellar extension, hypo-intensity on T1-weighted imaging, hyperintensity on T2-weighted imaging, and peripheral rim enhancement following gadolinium injection (figure 1/A). The blood sample workup revealed WBC $18950 \mu \mathrm{L}$ (normal 4000 - 10000), hemoglobin $12.9 \mathrm{~g} / \mathrm{dL}$, platelets $617000 / \mu \mathrm{L}$ (normal 150000 - 450000); CRP 313mg/L (normal $\leq 6$ ). Endocrine investigations were normal.

Table 1 Follow-up of biologic parameters

\begin{tabular}{|l|l|c|c|c|c|c|c|c|c|}
\hline & \multicolumn{5}{|c|}{ Blood } & \multicolumn{5}{c|}{ CSF } \\
\hline & WBC & Hb(g/d) & Platelet & CRP (mg/l) & Protein $(\mathbf{m g} / \mathbf{m l})$ & Glucose (g/l) & WCC & RBC & Culture \\
\hline Day 0 & 18950 & 12.9 & 617000 & 313 & & & & & \\
\hline Day 6 & 16740 & 11.1 & 412000 & 128 & 2.83 & 0.29 & 140 & 50 & negative \\
\hline Day 16 & 7040 & 12.8 & 418000 & 11.03 & 0.50 & 0.45 & $<10$ & 2 & negative \\
\hline Day 21 & & & & 7.2 & & & & & \\
\hline
\end{tabular}

WBC: white blood cell; Hb: hemoglobin; CRP: C-reactive protein; WCC: white cell count; CSF: cerebrospinal fluid
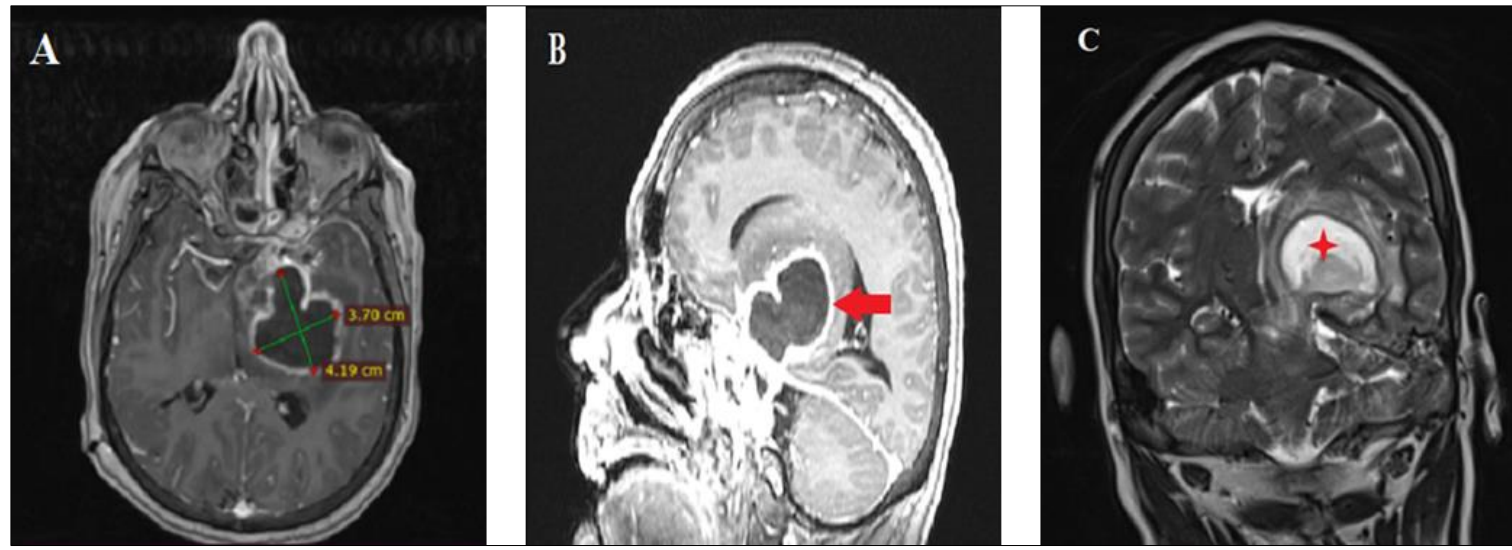

Figure 1 (A) Axial T1-weighted image showing a 37X41.9 mm lesion enhanced at the margin after gadolinium injection. (B) T1-weighted sagittal image shows hypointense enhancing after gadolinium injection with suprasellar extension (red arrow). (C) Coronal T2-weighted image shows the lesion in hyperintensity (red star)
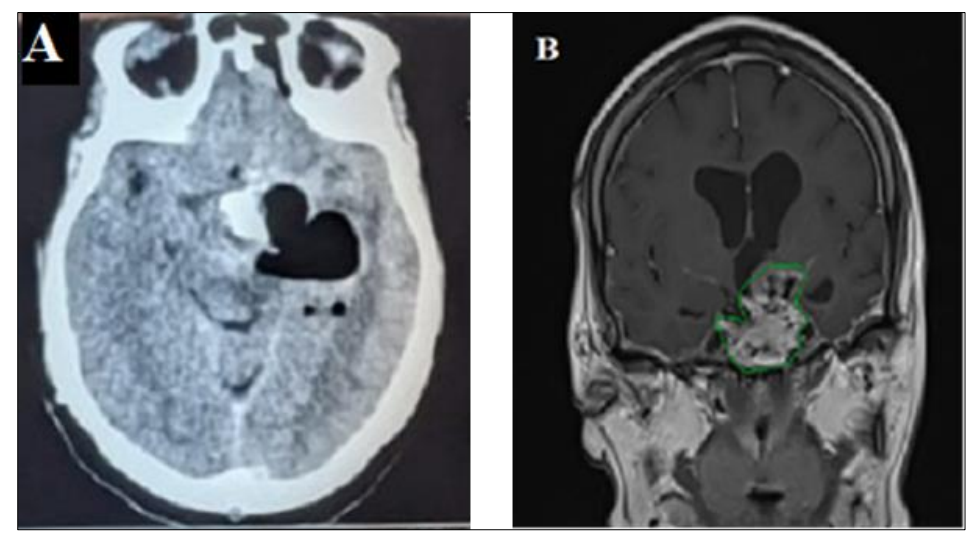

Figure 2 (A) Axial brain CT scan image after evacuation shows an emptying of the intratumoral collection. (B) Coronal T1-weighted image of control six months later 
The patient underwent stereotactic evacuation of the fluid. It was an abscessed, yellow-brownish, thick, and malodorous collection. The fluid analysis was sterile and non-pathogen was identified. A follow-up brain CT scan was performed, confirming a complete evacuation of the abscess (Figure 2/A). Cerebrospinal fluid (CSF) analysis was performed because of the persistence of fever five days later after administration of antibiotics; and revealed that protein 4.58 and WBC 140 mostly neutrophil polynuclear was increased, glucose 0.29 level decreased. Direct examination and culture were negative. Management was mainly medical, with broad-spectrum antibiotics ceftriaxone $6 \mathrm{~g}$ per day, Ciprofloxacin $800 \mathrm{mg}$ per day, and metronidazole $1.5 \mathrm{~g}$ per day for 6 weeks.

The clinical evolution was marked by a progressive neurological recovery, absence of fever, and normalization of the inflammatory biological parameters (table1). The patient was seen six months later for follow-up. Clinically, she retained hemiparesis. The control MRI performed did not show a recurrent abscess (Figure 2/B).

\section{Discussion}

Abscess secondary to a pituitary adenoma is rare and associated with high morbidity [4]. Apart from the endocrine signs that often accompany the clinical picture, the common neurological manifestations found in most series are headache, meningismus, and visual disturbances $[4,5,6,7]$. Most focal neurological deficits often involve the cranial oculomotor nerves [7]. These clinical presentations are also found in pituitary apoplexy and craniopharyngioma [8, 9]. To the best of our knowledge, only one case of pituitary abscess with hemiparesis was reported by Nelson in 1983 [10]. In our context, hemiplegia was associated with headache and fever. Thus, there is no specific clinical manifestation to put forward for the diagnosis of abscessed pituitary adenoma. Hence the importance of referring to radiological investigations to eliminate differential diagnoses.

Pituitary MRI is the gold standard imaging for the pituitary gland and the parasellar investigation. In pituitary abscess, MRI shows a sellar round cystic mass with or without suprasellar extension, slight hypo- or isointense on T1-weighted imaging, iso- or hyperintensity on T2-weighted imaging, and peripheral rim enhancement following gadolinium injection [7, 11, 12]. This appearance on MRI is non-specific [12].

Because of non-specific clinical features and imaging findings, preoperative diagnosis of a pituitary abscess is difficult $[13,14,15]$. However, the presence of a biological inflammatory syndrome suggests a pituitary abscess [15]. In most cases, the diagnosis of abscess pituitary is intraoperative [4], and then followed by the analysis of the abscess material. Microbiologic and histologic investigations are gold standards of diagnosis [5]. Thirteen percent of the patients in the Gao et al series had a positive culture [7]. Other authors reported a negative culture in half the cases [4]. In our case the culture was negative. Authors have stated that a pituitary abscess in an adenoma is a normal tissue reaction to infarction of a pituitary adenoma [10].

There are implicated risk factors for the occurrence of abscesses in a pituitary adenoma. Some factors leading to the development of an abscess in an adenoma include altered blood flow and areas of necrosis or local immunological alteration in the adenoma [14]. In our case, it can be the pre-existing disorder on the pituitary gland (aggressive pituitary macroadenoma), invasion of the sphenoidal sinus, or exposure to sellar radiation. The hypothesis of an abscess secondary to surgical treatment should not be ruled out. Nelson and al reported the possibility of a distant abscess [10].

The management of a pituitary abscess relies mainly on surgical drainage and antibiotic treatment [15]. In most series, the transsphenoidal approach is unanimously approved [1-15]. The transcranial approach is recommended if the abscess is located in the suprasellar region or if the suprasellar extent of the abscess is so large that the transsphenoidal approach is unlikely to allow significant evacuation of the abscess and decompression of suprasellar structures [5]. In our setting, we proceeded with a stereotactic evacuation to ensure surgical drainage and decompression. This minimally invasive approach was performed to minimize brain damage because of the deep localization of the abscess. Stereotactic aspiration has several advantages and can be repeated safely [16].

Antibiotic treatment is an essential part of the management of a pituitary abscess. It should be started without delay if a pituitary abscess is suspected. In most series, antibiotic therapy was adapted to the pathogen [4-7]. In our case, the empirical antibiotic treatment was early.

The outcome was favorable in our case, although mortality is high in pituitary abscesses [7]. 


\section{Conclusion}

Pituitary abscess secondary to an adenoma is rare. It can be accompanied by serious neurological signs. Thus, it must be diagnosed early because it is a serious complication.

\section{Compliance with ethical standards}

\section{Disclosure of conflict of interest}

The authors did not receive any funding for the preparation of this case report.

\section{Statement of informed consent}

Informed consent and verbal permission were obtained from the patient and her family before the submission of this article. In addition, this article follows both the Consensus-based Clinical Case Reporting Guideline and the Recommendations for the Conducting, Reporting, Editing, and Publication of Scholarly Work in Medical Journals.

\section{References}

[1] Swearingen B, Biller BM. Diagnosis and management of pituitary disorders. Springer Science \& Business Media: Humana Press; 2008.

[2] Raverot G, Burman P, McCormack A, Heaney A, Petersenn S, Popovic V, Trouillas J, Dekkers OM. European Society of Endocrinology Clinical Practice Guidelines for the management of aggressive pituitary tumors and carcinomas. Eur J Endocrinol. 2018; 178: G1-G24.

[3] Safaee MM, Blevins L, Liverman CS, Theodosopoulos PV. Abscess Formation in a Nonfunctioning Pituitary Adenoma. World Neurosurg. juin 2016; 90: 703.e15-703.e18.

[4] Hatiboglu MA, Celal Iplikcioglu A, Ozcan D. Abscess formation within invasive pituitary adenoma. J Clin Neurosci. Août. 2006; 13(7): 774-7.

[5] Zhang X, Yu G, Du Z, Tran V, Zhu W, Hua W. Secondary pituitary abscess inside adenoma: A case report and review of literature. World Neurosurg. 2020; 137: 281-5.

[6] Liu Y, Liu F, Liang Q, Li Y, Wang Z. Pituitary abscess: report of two cases and review of the literature. Neuropsychiatr Dis Treat. 2017; 13: 1521.

[7] Gao L, Guo X, Tian R, Wang Q, Feng M, Bao X, et al. Pituitary abscess: clinical manifestations, diagnosis and treatment of 66 cases from a large pituitary center over 23 years. Pituitary. Avr. 2017; 0(2): 189-94.

[8] Barkhoudarian G, Kelly DF. Pituitary apoplexy. Neurosurg Clin. 2019; 30(4): 457-63.

[9] Bhaisora KS, Prasad SN, Das KK, Lal H. Abscess inside craniopharyngioma: diagnostic and management implications. BMJ Case Rep. 3 févr. 2018; bcr-2017-223040.

[10] Nelson PB, Haverkos H, Martinez JA, Robinson AG. Abscess Formation within Pituitary Tumors. Neurosurgery. 1 mars. 1983; 12(3): 331-3.

[11] Wang Z, Gao L, Zhou X, Guo X, Wang Q, Lian W, et al. Magnetic resonance imaging characteristics of pituitary abscess: a review of 51 cases. World Neurosurg. 2018; 114: e900-12.

[12] Bladowska J, Sokolska V, Czapiga E, Badowski R, Koźmińska U, Moroń K (2004) Advances in diagnostics imaging of the pituitary and the parasellar region. Adv Clin Exp Med. 2004; 13: 709-717.

[13] Kuge A, Sato S, Takemura S, Sakurada K, Kondo R, Kayama T. Abscess formation associated with pituitary adenoma: A case report: Changes in the MRI appearance of pituitary adenoma before and after abscess formation. Surg Neurol Int. 2011; 2.

[14] Vates GE, Berger MS, Wilson CB. Diagnosis and management of pituitary abscess: a review of twenty-four cases. J Neurosurg. 2001; 95(2): 233-41.

[15] Su Y-H, Chen Y, Tseng S-H. Pituitary abscess. J Clin Neurosci. 2006; 13(10): 1038-41.

[16] Boviatsis EJ, Kouyialis AT, Stranjalis G, Korfias S, Sakas DE. CT-guided stereotactic aspiration of brain abscesses. Neurosurg Rev. juill. 2003; 26(3): 206-9. 